\title{
LKB1, a Key Driver Gene of Human Lung Squamous Cell Carcinoma
}

\author{
Wei Jiang and Qitao Yu* \\ Medical Oncology of Respiratory, Affiliated Tumor Hospital of Guangxi Medical University, Nanning, China
}

*Corresponding author: Qitao Yu, Medical Oncology of Respiratory, Affiliated Tumor Hospital of Guangxi Medical University,

71 Hedi Road, Qingxiu District, Nanning, China

\begin{tabular}{|c|c|}
\hline ARTICLE INFO & ABSTRACT \\
\hline Received: 蔧 July 01, 2019 & Citation: Wei Jiang, Qitao Yu. LKB1, a Key Driver Gene of Human Lung Squamous Cell \\
\hline Published: 蔧 July 08, 2019 & a. Biomed J Sci \& Tech Res 19(3)-2019. BJSTR. MS.ID.003302. \\
\hline
\end{tabular}

\section{Introduction}

Identification of driver genes not only is a challenging work for cancer study, including lung cancer, but also is a foundation of precision medicine [1]. For example, the discovery of driver genes (e.g. EGFR, ALK and ROS1) in lung adenocarcinoma led to the development of molecular targeted therapies, significantly improving the treatment outcome. Unfortunately, similar targeted therapies have not been developed for the treatment of lung squamous cell carcinoma (LSCC), accounting for approximately 25$30 \%$ lung cancer patients. This may be due to a lack of evidence showing the key drivers of LSCC. Unlike that overexpression of single EGFR mutation in mice can induce lung adenocarcinoma, none of individual highly mutated genes in human LSCC tumors has been found to induce LSCC in vivo [2]. Therefore, the unaddressed key driver genes of LSCC prevent from developing its targeted therapy. Recently, Jian Liu et al. reported that $L k b 1$ ablation alone in mouse lungs induced LSCC, providing the direct in vivo evidence of showing $L k b 1$ to be the key driver gene of LSCC [3].

Although Lkb1 has been reported to involve in LSCC development under another genetic background, such as $\mathrm{Kras}^{\mathrm{G} 12 \mathrm{D}}$ [4], Sox2 [5], and Pten [6], the role of Lkb1 in determining LSCC development has been undermined since no phenotype was found after ablation of $L k b 1$ using viral Cre [4-6]. Considering the potential limit of the viral Cre, Jian Liu et al. generated a Cre mouse, named CCSPicre [7], to examine several candidate genes in their study. The advantage of this Cre is reported to have strong activity in the large airway epithelium, a major cell population responsible for human LSCC development. Notably, LSCC tumors were induced only after ablation of $L k b 1$ using this Cre, whereas manipulation of other five frequent genetic mutations ( $p 53$, Pten, Errfi1, Smad4, and Kras ${ }^{\mathrm{G} 12 \mathrm{D}}$ ) alone is unable to generate LSCC. This not only demonstrates $L k b 1$ to be a key suppressor gene in LSCC initiation and progression but also suggest this Cre mouse line as a valuable tool to explore the de novo functions of LSCC regulators.

Mechanistically, Jian Liu et al. showed that $L k b 1$ deficiency resulted in decreased expression of MKK7, a reduction of JNK1/2 phosphorylation, lower JNK1/2 activities and elevated $\Delta$ Np63 signaling, which subsequently led to initiation and progression of LSCC in vivo. Moreover, the authors revealed that the secondary genetic alterations beyond $L k b 1$ alternation might be necessary for stimulation of LSCC formation. For example, Jnk1/2 loss accelerated Lkb1-null induced LSCC development and JNK1/2 activation attenuated mouse LSCC development. Therefore, more genetic regulators of LSCC are expected to be identified in a combination of $L k b 1$ deletion. Clinically, Jian Liu et al. also reported that JNK1/2 was inactivated in a large proportion of LSCC patients and higher JNK1/2 activities had a better survival rate and longer relapse-free survival. JNK1/2 activators, such as Anisomycin or its derivatives, might benefit LSCC patients with low JNK1/2 activities through simulation of JNK1/2 signaling. Interestingly, JNK1/2 activities also exhibited a positive relationship with the survival rate of the cervical or head and neck SCC patients. Thus, activation of JNK1/2 in LSCC patients with lower JNK1/2 gene signature will be attractive clinical trials.

Besides, several LSCC mouse models generated in this study (e.g

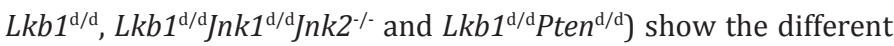
stages of LSCC development, including the initial hyperplasia and squamous metaplasia. Further investigation of these development stages of LSCC using single cell sequence can help identify the 
cellular origins and the track of genetic evolutionary dynamics during LSCC development. Despite this progress, it is noteworthy that LSCC tumors in an advanced stage, even in early stage, have complex genetic alterations other than one single genetic disorder. This dysregulated genetic complex poses a tremendous challenge to effectively provide LSCC targeted treatment. For example, tyrosine kinase inhibitors (e.g. Taselisib targeting PI3K, Palbociclib targeting cell cycle gene alteration, and AZD4547 targeting FGFR) have been specifically applied in treating human LSCC tumors with the related genetic alternations, but the outcome is disappointed, evidenced by the low response rates $(<7 \%)$ and median progression-free survival time (2.9, 1.7 and 2.7 months, respectively) [8-10]. Therefore, identification of more regulators of LSCC besides the key drivers (e.g. $L k b 1$ ) merits the urgent further investigation, which can significantly help develop the effective combination of targeted therapy and bring the clinical benefit to LSCC patients.

\section{References}

1. Herbst RS, Morgensztern D, Boshoff C (2018) The biology and management of non-small cell lung cancer. Nature 553(7689): 446-454

2. Singh AP, Adrianzen Herrera D, Zhang Y, Perez Soler R, Cheng H (2018) Mouse models in squamous cell lung cancer: impact for drug discovery. Expert Opin Drug Discov 13(4): 347-358.

3. Liu J, Wang T, Creighton CJ, Wu SP, Ray M, et al. (2019) JNK(1/2) represses Lkb(1)-deficiency-induced lung squamous cell carcinoma progression. Nat Commun 10(1): 2148.

\section{ISSN: 2574-1241}

DOI: $10.26717 /$ BJSTR.2019.19.003302

Qitao Yu. Biomed J Sci \& Tech Res

This work is licensed under Creative Commons Attribution 4.0 License

Submission Link: https://biomedres.us/submit-manuscript.php
4. Ji H, Ramsey MR, Hayes DN, Fan C, McNamara K, et al. LKB1 modulates lung cancer differentiation and metastasis. Nature 448(7155): 807-810.

5. Mukhopadhyay A, Berrett KC, Kc U, Clair PM, Pop SM, et al. Sox cooperates with Lkb1 loss in a mouse model of squamous cell lung cancer. Cell Rep 8(1): 40-49.

6. Xu C, Fillmore CM, Koyama S, Wu H, Zhao Y, et al. (2014) Loss of Lkb1 and Pten leads to lung squamous cell carcinoma with elevated PD-L1 expression. Cancer Cell 25(5): 590-604.

7. Liu J, Cho SN, Akkanti B, Jin N, Mao J, et al. (2015) ErbB2 Pathway Activation upon Smad4 Loss Promotes Lung Tumor Growth and Metastasis. Cell Rep 10(9): 1599-1613.

8. Langer CJ, Redman MW, Wade JL, Aggarwal C, Bradley JD, et al. (2019) Brief Report: SWOG S1400B (NCT02785913), A Phase II Study of GDC0032 (Taselisib) for Previously Treated PI3K-Positive Patients with Stage IV Squamous Cell Lung Cancer (Lung-MAP Sub-Study). J Thorac Oncol.

9. Aggarwal C, Redman MW, Lara P, Borghaei H, Hoffman PC, et al. (2017) Phase II study of the FGFR inhibitor AZD4547 in previously treated patients with FGF pathway-activated stage IV squamous cell lung cancer (SqNSCLC): LUNG-MAP sub-study SWOG S1400D. Journal of Clinical Oncology 35(15Suppl): 9055.

10. Edelman MJ, Redman MW, Albain KS, McGary EC, Rafique N, et al. (2017) A phase II study of palbociclib (P) for previously treated cell cycle gene alteration positive patients (pts) with stage IV squamous cell lung cancer (SCC): Lung-MAP sub-study SWOG S1400C. Journal of Clinical Oncology 35(15Suppl): 9056 